\title{
Association between Heights of Dialysis Patients and Outcomes: Results from a Retrospective Cohort Study of the International MONitoring Dialysis Outcomes (MONDO) Database Initiative
}

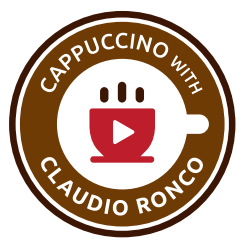

\author{
Samir Patel ${ }^{\mathrm{a}}$ Alice Topping ${ }^{\mathrm{a}}$ Xiaoling Ye $^{\mathrm{a}}$ Hanjie Zhang $^{\mathrm{a}}$ Bernard Canaud $^{\mathrm{b}}$ \\ Paola Carioni $^{b}$ Cristina Marellic ${ }^{c}$ Adrian Guinsburg ${ }^{c}$ Albert Power ${ }^{d, e}$ \\ Neill Duncan ${ }^{d}$ Jeroen Kooman ${ }^{f}$ Frank van der Sande ${ }^{f}$ Len A. Usvyat ${ }^{a, g}$ \\ Yuedong Wang $^{\mathrm{h}}$ Xiaoqi Xu ${ }^{\mathrm{i}}$ Peter Kotanko ${ }^{\mathrm{a}}{ }^{\mathrm{j}}$ Jochen G. Raimann ${ }^{\mathrm{a}}$ and the \\ MONDO Initiative
}

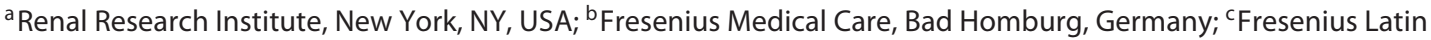

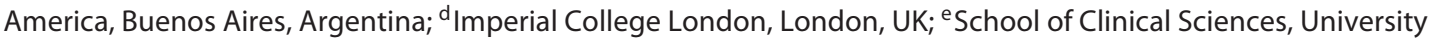
of Bristol, Bristol, UK; ${ }^{f}$ Maastricht University Medical Center, Maastricht, The Netherlands; ${ }^{9}$ Fresenius Medical Care,

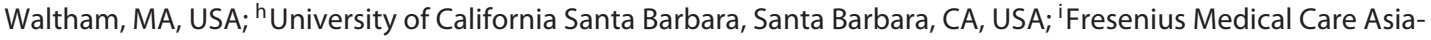
Pacific, Hong Kong, PR China; 'jlcahn School of Medicine at Mount Sinai, New York, NY, USA
\end{abstract}

\section{Keywords}

Height · Outcomes · Dialysis · Intercontinental databases · Monitoring dialysis outcomes

\footnotetext{
Abstract

Background: Tall people have improved metabolic profiles and better cardiovascular outcomes, a relationship inverted in dialysis patients. We investigated the relationship between height and outcomes in incident hemodialysis (HD) patients commencing treatment in an analysis of the international Monitoring Dialysis Outcomes (MONDO) database. Methods: In this retrospective cohort study, we included incident HD patients commencing treatment between January 1, 2006 and December 31, 2010 and investigated the association between height and mortality using the MONDO database. A 6-months baseline period preceded 2.5 years of follow-up, during which we recorded patient mortality. Patients were stratified in region-specific deciles of the respec-
}

tive database's population (Asia Pacific, North and South America, and Europe) and we developed Cox-proportional hazard models (additionally adjusted for age, gender, postdialysis weight, eKt/V, albumin, interdialytic weight gain, phosphorus, and predialysis systolic blood pressure) for each database. Results: We studied 23,353 patients (62 \pm 15 years old, $42 \%$ female, body mass index $26 \pm 6 \mathrm{~kg} / \mathrm{m}^{2}$, height $165 \pm 10 \mathrm{~cm})$. We found a trend of increasing hazard ratio of death (HR) with increasing height for Asia Pacific, Europe, and South America. In the fully adjusted models, for South America, we found a trend of increasing HR without significance among deciles $>5$. In Europe, deciles 8-10 had significantly increased HR. No clear trend was found in North America. Conclusion: We found an increasing risk of death with increasing height in all regions, except North America. While the reasons remain unclear, further research may be warranted.

Video Journal Club 'Cappuccino with Claudio Ronco' at http://www.karger.com/?doi=485162. @ 2018 S. Karger AG, Basel
(C) 2018 S. Karger AG, Base

E-Mail karger@karger.com

www.karger.com/bpu
Jochen G. Raimann, MD, $\mathrm{PhD}$

Renal Research Institute

315 E 62 nd St, 4TH Floor

New York, NY 10065 (USA)

E-Mail jochen.raimann@rriny.com 


\section{Introduction}

Height in adults varies greatly and average population heights also vary between the different regions of the world. For example, shorter heights are more common in Asia Pacific as compared to North America and Europe. In Europe, height has been increasing for men and women over the decades. As an example, in Italy in 1950, the average height of men was $172.5 \mathrm{~cm}$, and for women it was $161.4 \mathrm{~cm}$. In 1980 it was $177.1 \mathrm{~cm}$ for men and 166.5 $\mathrm{cm}$ for women [1]. Achieved height is multifactorial, but is largely attributable to genetic determination, which is evidenced by an association between the height of parents and their offspring in adulthood. The increase in height over decades is likely epigenetically determined [2].

Height has a direct relationship with outcomes in the general population. Taller people have greater diameters of coronary arteries, and this results in less plaque burden and therefore lower rates of coronary artery disease [3]. Recent studies have shown that taller people are at a greater risk for thromboembolic events, possibly due to having greater venous surface areas, and more venous valves [4]. In addition, shorter people have an increased risk of cardiovascular disease (CVD) and have a worse lipid profile [5]. Shorter people also have more risk factors for CVD such as higher blood pressure, higher levels of low density lipoprotein cholesterol, and diabetes.

A retrospective cohort was done in the United States with 1,171,842 patients to evaluate the relationship between height and outcomes in dialysis patients. Patients were divided into quintiles and mortality risk was studied based on race and gender. It was found that for men and women, the hazard ratio (HR) increased among quintiles. On the basis of race, for black men and women, the risk did not increase among quintiles [6]. In another study, a cohort of 117,644 maintenance hemodialysis (HD) patients was created, and a prospective study was done to look at height and outcomes, while also looking at body mass index (BMI). It was found that height and weight were directly associated with all-cause mortality [7].

In order to determine if height is associated with outcomes, and whether this relationship is consistent in different regions, we investigated the relationship between height and outcomes in the international Monitoring Dialysis Outcomes (MONDO) database, which consists of data from North America, South America, Europe, and Asia Pacific. To our knowledge, no other study has investigated this relationship on the global level. We hypothesize that an association between height and risk of death in $\mathrm{HD}$ patients will be present in all regions.

\section{Methods}

We studied incident patients commencing HD between January 2006 and December 2010 in the MONDO initiative. The initiative comprises HD databases from Renal Research Institute clinics in the United States; Fresenius Medical Care (FMC) clinics in Europe, Asia Pacific, and South America; KfH clinics in Germany; and clinics associated with the Imperial College (United Kingdom), Hadassah Medical Center (Israel), and Maastricht University Medical Center (The Netherlands). The data are grouped into four regional categories: North America (NA), South America (SA), Europe (EU), and Asia Pacific (AP), identifying information was entirely removed [8]. These de-identified electronic medical records are then incorporated into a primary database and used for analysis [9].

We studied the association between height and overall risk of mortality. Height in centimeters was recorded for each patient at the initiation of dialysis. Height was analyzed as a categorical variable. Patients were stratified into deciles of height by gender in the respective regions. Deciles were derived in a similar fashion to a previous paper by Shapiro et al. [7] in order to make direct comparisons of this international cohort to the large US population studied in that paper. We then analyzed the risk of death for each height decile, utilizing a 6-month baseline and 2.5-year follow-up. Of the original 32,779 patients in our dataset, those with incomplete data were excluded from our cohort, and the remaining 23,353 patients were included in the study.

\section{Statistical Analysis}

Measurements of central tendency were reported for continuous metrics using the mean and SD for normally distributed data and median and interquartile range for non-normally distributed data. Frequencies and percentages for each level of the variable are reported for categorical or binary metrics. A Cox proportional hazards $(\mathrm{PH})$ model was developed to understand the effect of height on hazard of death. We created 3 Cox PH models to assess the risk of death by height decile while taking into account the time to death. In the first model, we adjusted for age, gender, and post-dialysis weight. The next model included the previous adjustments and added an adjustment for eKt/V. The fully adjusted model included adjustments for age, gender, post-dialysis weight, eKt/V, albumin, inter-dialytic weight gain (IDWG; kg), phosphorus, and pre-dialysis systolic blood pressure (SBP). Decile 5 was used as the reference group. Sex-standardized height deciles were analyzed in the 4 regions separately with all 3 models to assess the differences in each region. Analysis was performed using SAS 9.4 (SAS Institute, Cary, NC, USA). Height deciles 4 and 5 in North American women were of the same height of $160 \mathrm{~cm}$ due to the small sample size.

\section{Results}

We studied 23,353 patients who started HD between January 1, 2006 and to December 31, 2010. Half of the patients were from Europe, while 25\% were from North America, 14 and 11\% were from Asia Pacific and South 
Fig. 1. Male and female height distribution per region. Asia Pacific: Australia, Hong Kong, South Korea, Malaysia, Philippines, Singapore, Turkey, Taiwan, Thailand. Europe: Bosnia, Czech Republic, France, Hungary, Ireland, Italy, Poland, Portugal, Romania, Russia, Serbia, Slovakia, Slovenia, Spain, United Kingdom. North America: United States. South America: Argentina, Brazil, Olile, Columbia.

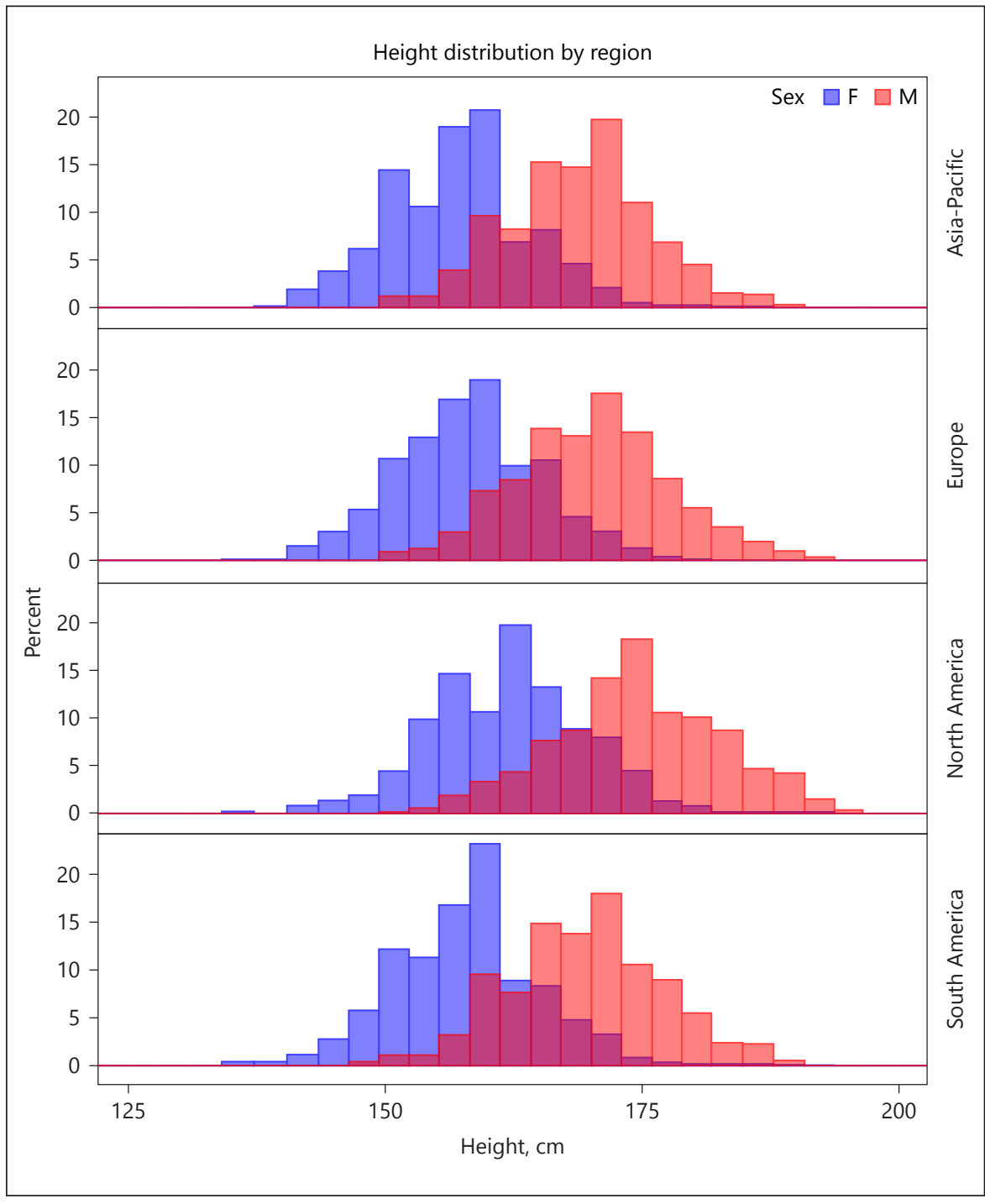

America respectively. The mean age was $62 \pm 15,41 \%$ were female, average BMI was $26 \pm 6 \mathrm{~kg} / \mathrm{m}^{2}$, and the mean height was $165 \pm 10 \mathrm{~cm}$. The mean heights at the start of dialysis in each continent were as follows: North America, $168.3 \pm 10.8 \mathrm{~cm}$; SA, $164 \pm 9.5 \mathrm{~cm}$; Europe, $164.7 \pm 9.8 \mathrm{~cm}$; and AP, $162.9 \pm 9.3 \mathrm{~cm}$. Dialysis patients were slightly taller on average in North America. Average height differed by sex in each region with men on average being taller than women (overall men $170 \pm 8.4 \mathrm{~cm}$ vs. overall women $158 \pm 7.7 \mathrm{~cm}$; Fig. 1).

Overall, $59 \%$ of the overall sample consisted of men, and all height deciles contained a majority of men. $\mathrm{Pa}$ tients in the tallest height decile were younger at an age of $55.9 \pm 15.6$ and age increased at each descending height decile to $67.5 \pm 14.9$ years in the shortest height decile
(Table 1a). In our cohort, patients in Asia were on average shorter than in the other regions (Table 1b). Average postdialysis weight increased as height decile increased (61.5 \pm $13.9 \mathrm{~kg}$ in height decile $1 \mathrm{vs.} .85 \pm 22 \mathrm{~kg}$ in height decile 10 ). BMI remained nearly constant across height deciles with an overall BMI of $26.4 \pm 5.7 \mathrm{~kg} / \mathrm{m}^{2}$ and no discernable trend by height decile. However, eKt/V decreased incrementally from $1.48 \pm 0.29$ in height decile 1 to $1.29 \pm 0.26$ in height decile 10. Phosphorus increased slightly by height decile, starting at an average of $4.7 \pm 1.1 \mathrm{mg} / \mathrm{dL}$ in height decile 1 and increasing to $5.1 \pm 1.2 \mathrm{mg} / \mathrm{dL}$ in decile 10. Albumin remained nearly constant across all height deciles at $3.8 \pm 0.4 \mathrm{~g} / \mathrm{dL}$. Pre-treatment sitting SBP varied slightly among groups, but with no clear trend, while IDWG in kilograms increased slightly with height decile. 

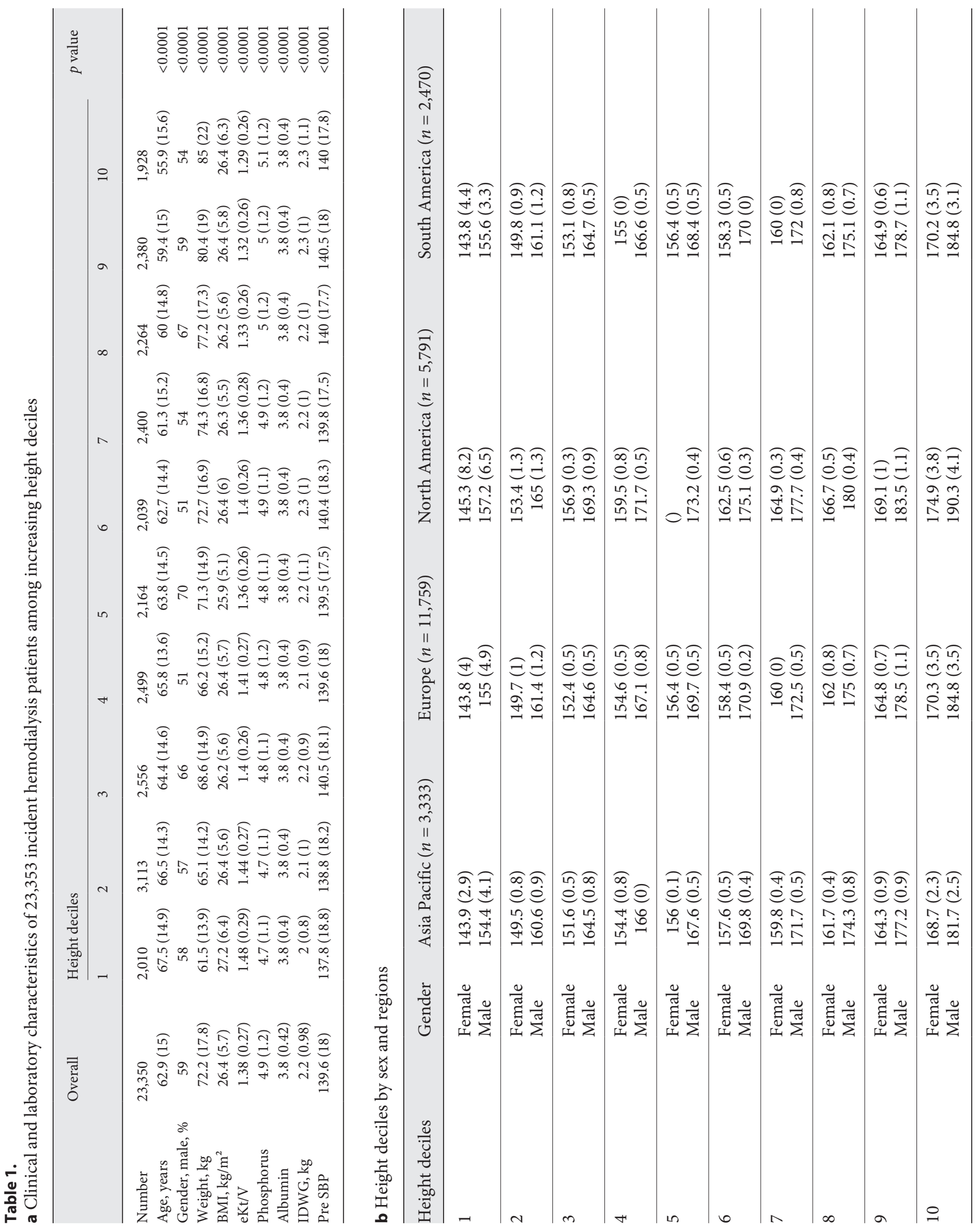
Fig. 2. a-c Forest plots of height deciles and adjusted hazard ratio of all-cause death with adjustments. All models included incident patients, a 6 months baseline, a follow-up period of 2.5 years.

(For figure $2 b$, c see next pages.)

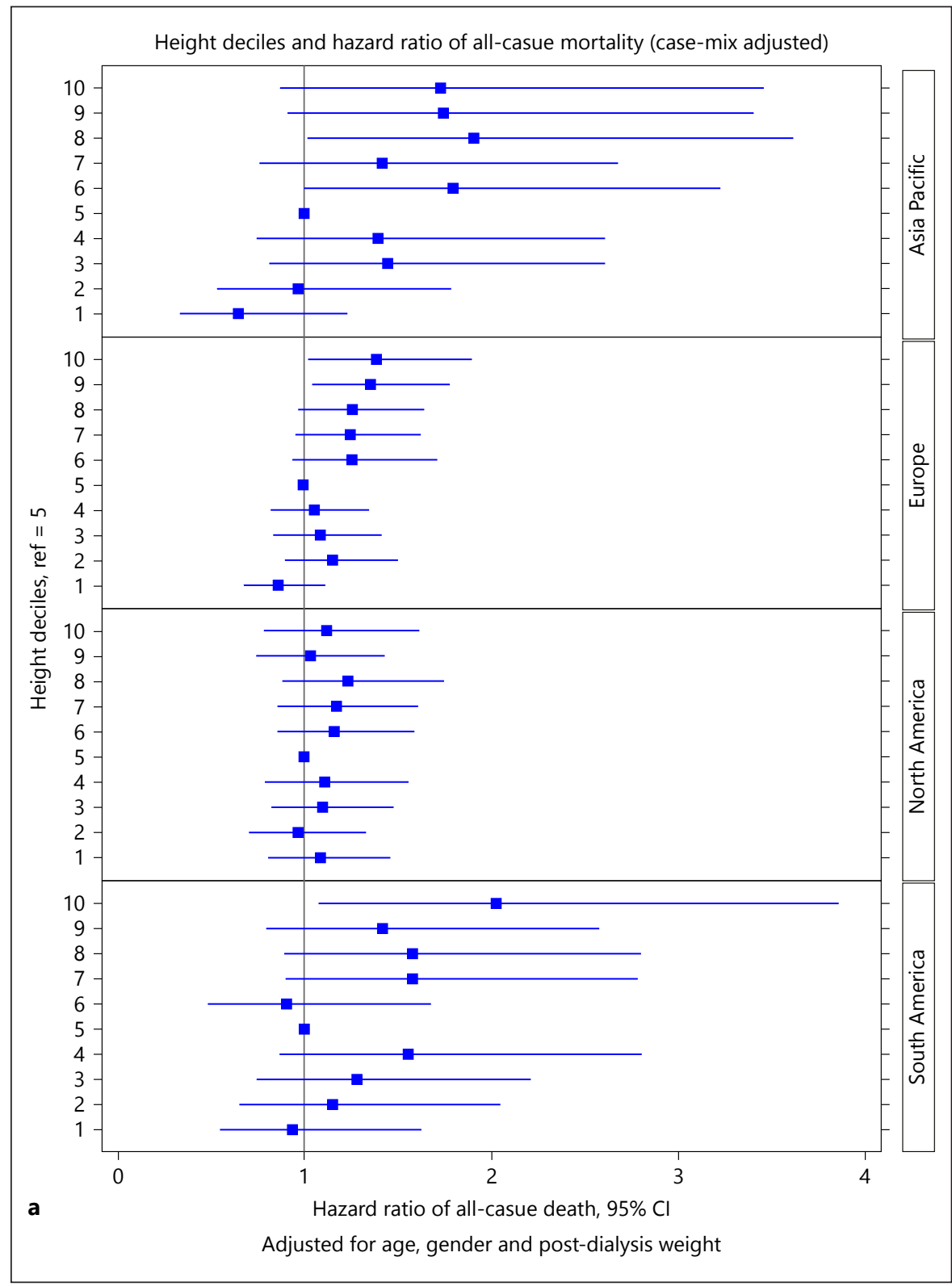

There were 2,393 deaths and the crude event rate during the follow-up period was $10.2 \%$. The event rate varied by region in the following manner: $7.6 \%$ in Asia Pacific, 8.8\% in Europe, $10.6 \%$ in South America and $14.6 \%$ in North America. Results from the Cox PH model show a discernable trend of increasing HR of death with increasing height decile in Asia Pacific, Europe, and Latin America when adjusting for age, post-dialysis weight, eKt/V, albumin, IDWG, phosphorus, and pre-treatment SBP (Fig. $2 \mathrm{a}-\mathrm{c})$. No trend was found in North America. The results remained consistent across all three models. While most HRs did not reach significance, patients in the tallest height deciles in South America and Europe were found to be at greater risk of death with an HR of 1.4 (95\% CI 1.02-1.9) in Europe height decile 10, 1.3 (95\% CI 1.01-1.8) in Europe height decile 9, and 1.9 (95\% CI 1.02-3.7) in South America height decile 10 when compared to the 5th height decile. We did not find a trend for risk of death among patients in shorter height deciles (Fig 2a-c). We also stratified as quintiles in the sensitivity analysis, and the results were the same as in the deciles. In our analysis, we adjusted for eKt/V and this renders the association robust even if considering treatment time. 


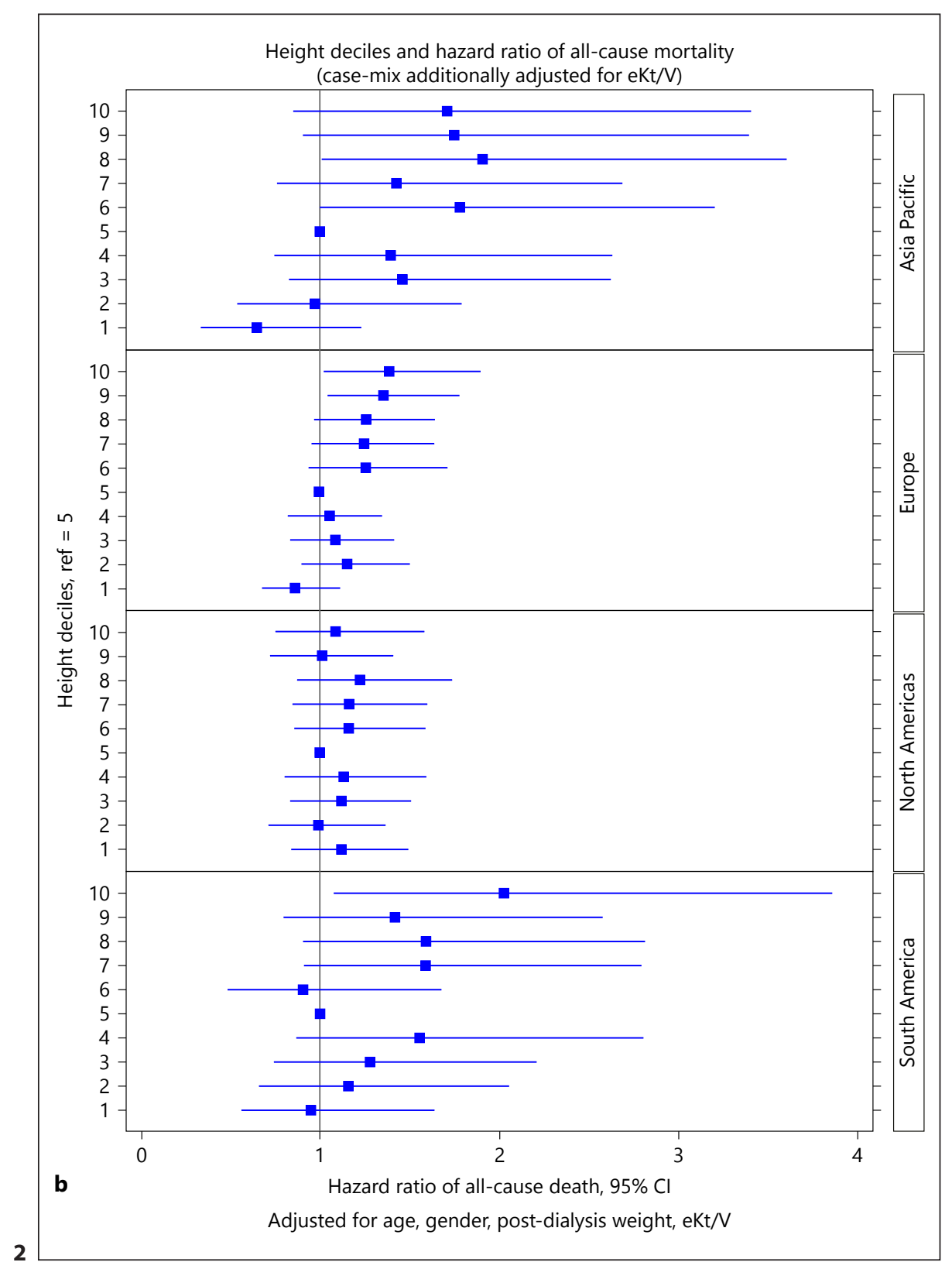

\section{Discussion}

Our analysis has shown that taller height carries a greater risk of mortality in HD patients in Asia Pacific, Europe, and South America. This was confirmed with statistical significance in South America and Asia Pacific. No clear discernible trend was observed in North America. Possible explanations for this include the fact that in North America, treatment times are much shorter than other regions of the world. In our subset analysis, we compared white and black patients in North America, and found no trend among the black patients. There was a general increasing trend among white patients. The trends we observed were consistent among the 3 models we implemented. The case mixed model, adjusted for age, gender, and post-dialysis weight, followed by the model including these adjustments plus eKt/V, and lastly, the fully adjusted model, additionally including albumin, IDWG, phosphorus, and pre-dialysis SBP. Our observation has shown evidence that $\mathrm{HD}$ patients have a different trend in the risk of adverse outcomes as compared to the general population, in which there is a decreasing risk of 


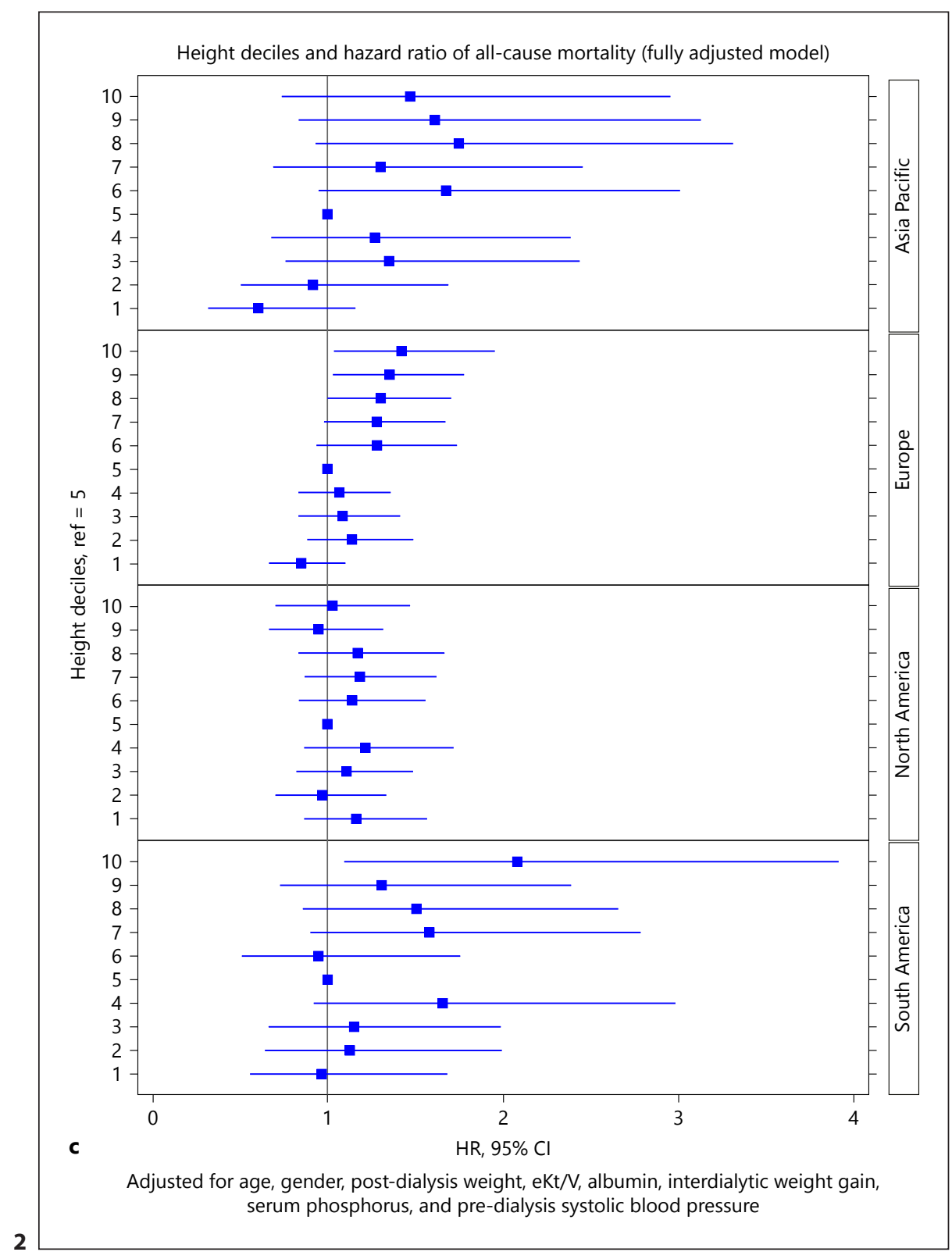

mortality with increasing height [10]. During our study, there were no changes in the heights of patients documented. The prevalence of certain comorbidities causing end-stage renal disease (ESRD), such as diabetes mellitus is variable in different regions of the world. In the United States, diabetes mellitus has a much higher rate of causing ESRD than in other countries [11]. Removing genderspecific height differences, the percent of men and women in the model are representative of their percentage in their gender-specific populations. In line with the study by Shapiro, we found an overall increase in HR with increasing height deciles.
Factors besides the genetic determination of height could be nutrition, external factors such as socioeconomic status, disposition to stress (mother, child, or juvenile), and maternal or juvenile smoking. Poor nutrition can lead to developmental delays and growth defects. If proper quantities of nutrients are not obtained from the diet, one is considered to be malnourished. In most parts of the world, this involves undernourishment due to poverty, lack of access to food, poor hygiene, or lack of care. This results in inadequate growth, and therefore, shorter heights. In this context, adequate or overnutrition could be responsible for the taller height of the population in 
North America, a region known for overnutrition. In North America, many individuals tend to overconsume calories and have a surplus of the daily requirement, which is in contrast to developing regions or regions of less privileged socioeconomic status. Additionally, environmental influences such as socioeconomic status effect prenatal and postnatal growth, which propagates into an individual's adult height [12].

There are variations in the growth of children, which affect their adult height. Among these is "constitutional delay," where a child has delayed bone growth with respect to their chronologic age. During puberty, they have a catch up in growth, and reach their normal adult stature [13]. On the contrary, children with "familial short stature" have shorter adult height, which is also present in their parents [14]. Also, it is known that maternal smoking during pregnancy is strongly associated with low birthweight and smaller sized babies as expected per gestational age [15], and also beginning to smoke during the years of development can adversely affect body growth. Smoking affects the pituitary gland in the brain, which produces the growth hormone that is essential for growth [16]. In addition, smoking increases carbon monoxide in the blood, which lowers blood oxygen levels, and therefore limits the blood's ability to transfer vitamins and minerals required for building muscle tissue [17].

\section{Strengths}

Key to our analysis, we used deciles that are comparable to those used by Shapiro et al. [7]. This allows for validity in the comparison of the results we present, as compared to Shapiro et al. [7]. An additional strength of our study was generalizability among regions. The trend of an increasing hazard ratio of all-cause death with increasing height decile was present in all regions except North America. The trend remained present even after adjustment for treatment related factors including albumin, IDWG, phosphorus, and pre dialysis SBP. We utilized 6 months baseline period, twice as long as Shapiro, who utilized a 90-day baseline after the initial 90 days of HD. This allowed for treatment stabilization of the incident patients. Our follow-up period of 2.5 years was also longer than the 1 year used by Shapiro, allowing for the incorporation of changing treatment practices.

\section{Limitations}

Our sample size was 23,353 , which is much smaller than that utilized by Elsayed 1,171,842 patients, and Shapiro 117,644 patients. In our data, the largest patient pop- ulation was in Europe, in which we had data from 11,759 patients. In North America, our dataset only included 5,791 patients. This is a tremendously smaller sample than that used by Shapiro. This small sample size could have potentially played a role in the differences in outcomes we observed, as the larger study sample size allows for less variability. Due to the smaller sample in North America, the distribution of height was in such a way that height deciles 4 and 5 in North American women were the same height of $160 \mathrm{~cm}$. Thus, HRs comparing height decile 4-5 in North America can only be interpreted to show a difference in effect of height for men. To be considered, the geographic distribution of patients in our cohort may have varied from those studied by Shapiro. Of note, our documentation was less consistent than that of Shapiro and reported lesser comorbidities than their study.

\section{Clinical Implications}

Based on the findings, we are able to determine that there is an increased risk of mortality in HD patients with taller height, and this trend is uniform among continents. This trend allows for risk identification and stratification of patients. Perhaps it would be in the best interest of taller HD patients to weigh more deeply their comorbidities and risk factors as a means of more stringent healthcare regulation. It could be beneficial for taller patients to receive longer treatment times; however, further studies into this topic would be required. Studies have shown that patients with late referrals to nephrologist for ESRD care have worse outcomes than those who have better preESRD care [18]. Mortality and hospitalization among dialysis patients varies among countries. The DOPPS (Dialysis Outcomes and Practice Patterns Study) study has shown that within Europe, CVD-related hospitalizations for HD patients are higher in France and Germany than United Kingdom [19]. The findings of our study add important knowledge for developing future risk prediction models. Dialysis providers are given the task of evaluating patients at dialysis initiation and throughout their longterm care. Risk prediction models, which assess a patient based on the data we have presented, could possibly have some beneficial effect on the mortality of taller patients. In addition, the possible need of adjustment of dialysis dose for taller patients to achieve adequate treatment is an area to further look into.

\section{Future Research}

Our findings shed light on the need for conducting more research into the physiology and pathophysiology of mortality in both healthy subjects and the dialysis pop- 
ulation. Additional areas of future research pertain to the effects of different dialysis doses on patients, with respect to height, and if taller patients are receiving adequate dialysis doses based on Kt/V. Studies have shown some beneficial effect of increased Kt/V on mortality [20], suggesting the possibility that taller patients may indeed benefit from higher dialysis doses. Regardless of body size, patients in North America receive around $4 \mathrm{~h}$ of dialysis treatment per session, which is shorter than what patients receive in Europe and Asia [21].

\section{Conclusion}

Our study is the first to report the association between height and outcomes of dialysis patients on the global level. The clear trends we have observed indicate that taller patients have an increased risk of mortality in Europe, Asia Pacific, and Latin America. This trend was not observed in North America, possibly due to differences in treatment times or comorbidities.

\section{Acknowledgments}

No external funding was received. The results presented in this manuscript have not been published in whole or part, except in the abstract form, at the Spring Clinical Meetings of the National Kidney Foundation in Boston, Massachusetts, 2016, 53rd Congress of the ERA-EDTA in Vienna, Austria, May 2016, and was presented at the Kidney Week of the American Society of Nephrology November 2017 in New Orleans, Louisiana.

S.P., J.G.R., and P.K., initiated and designed the study. A.T. and X.Y. analyzed the data. B.C., C.M., A.G., X.X., A.P., N.D., J.K., F.S., P.C., L.A.U., and Y.W. provided scientific input in their fields of expertise. All authors contributed to the manuscript revision and improvement.

\section{Disclosure Statement}

Drs. Bernard Canaud and Peter Kotanko hold stock in FMC. The Renal Research Institute is a wholly owned subsidiary of FMC. All other authors do not have any potential financial conflicts of interest to declare.

\section{References}

1 Garcia J, Quintana-Domeque C: The evolution of adult height in Europe: a brief note. Econ Hum Biol 2007;5:340-349.

2 Benonisdottir S, et al: Epigenetic and genetic components of height regulation. Nat Commun 2016;7:13490.

3 O'Connor NJ, et al: Effect of coronary artery diameter in patients undergoing coronary bypass surgery. Northern New England Cardiovascular Disease Study Group. Circulation 1996;93:652-655.

4 Roetker NS, et al: Taller height as a risk factor for venous thromboembolism: a Mendelian randomization meta-analysis. J Thromb Haemost 2017;15:1334-1343.

5 Nelson CP, et al; Genetically determined height and coronary artery disease. N Engl J Med 2015;372:1608-1618.

6 Elsayed ME, Ferguson JP, Stack AG: Association of height with elevated mortality risk in ESRD: variation by race and gender. J Am Soc Nephrol 2016;27:580-593.

7 Shapiro BB, et al: Association of height with mortality in patients undergoing maintenance hemodialysis. Clin J Am Soc Nephrol 2015;10:965-974
8 Usvyat LA, et al: The MONitoring dialysis outcomes (MONDO) initiative. Blood Purif 2013;35:37-48.

9 Control, C.f.D. and Prevention, HIPAA privacy rule and public health. Guidance from CDC and the US Department of Health and Human Services. MMWR supplements, 2003. 52:p. 1.

10 Engeland A, et al: Height and body mass index in relation to total mortality. Epidemiology 2003;14:293-299.

11 Young EW, et al: The dialysis outcomes and practice patterns study (DOPPS): an international hemodialysis study. Kidney Int 2000; 57:S74-S81.

$12 \mathrm{Li} \mathrm{H}$, et al: Associations between prenatal and postnatal growth and adult body size and composition. Am J Clin Nutr 2003;77:1498-1505.

13 Crowne E, et al: Final height in boys with untreated constitutional delay in growth and puberty. Arch Dis Child 1990;65:1109-1112.

14 Goh LH, How CH, Ng KH: Failure to thrive in babies and toddlers. Singapore Med J 2016; 57:287-291.

15 Jaakkola JJ, Gissler M: Maternal smoking in pregnancy, fetal development, and childhood asthma. Am J Pub Health 2004;94:136-140.
16 Rohleder N, Kirschbaum C: The hypothalamic-pituitary-adrenal (HPA) axis in habitual smokers. Int J Psychophysiol 2006;59:236243

17 Petersen AM, et al: Smoking impairs muscle protein synthesis and increases the expression of myostatin and MAFbx in muscle. Am J Physiol Endocrinol Metab 2007;293:E843E848.

18 Stack AG: Impact of timing of nephrology referral and pre-ESRD care on mortality risk among new ESRD patients in the United States. Am J Kidney Dis 2003;41:310-318.

19 Rayner HC, et al: Mortality and hospitalization in haemodialysis patients in five European countries: results from the Dialysis Outcomes and Practice Patterns Study (DOPPS). Nephrol Dial Transplant 2004;19: 108-120.

20 Held PJ, et al: The dose of hemodialysis and patient mortality. Kidney Int 1996;50:550556

21 Saran R, et al: Longer treatment time and slower ultrafiltration in hemodialysis: associations with reduced mortality in the DOPPS. Kidney Int 2006;69:1222-1228. 